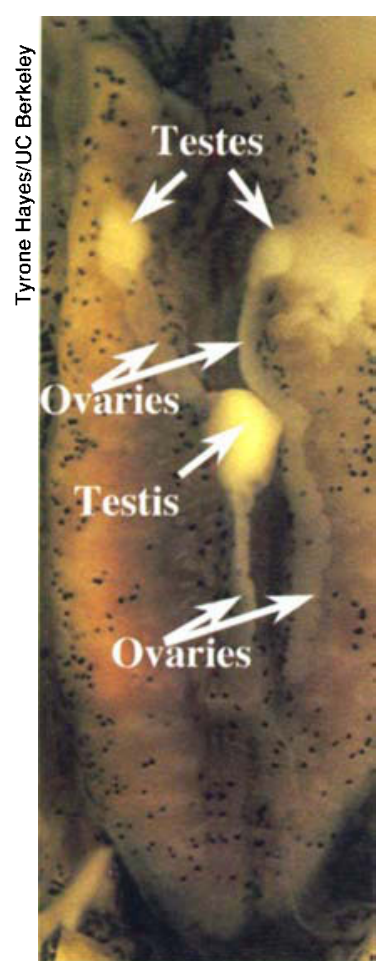

Abnormal gonads in a male Xenopus frog are the result of exposure to the herbicide atrazine. The frog has become a hermaphrodite, with both male (testes) and female (ovaries) sex organs.

\section{Atrazine scrambles frog sex organs}

Exposure to atrazine demasculinizes tadpoles and can turn them into hermaphrodites with both male and female sexual characteristics, UC Berkeley scientists have found.

In the April 16 Proceedings of the $\mathrm{Na}$ tional Academy of Sciences, developmental endocrinologist Tyrone Hayes and colleagues report that the widely applied herbicide also can lower levels of the male hormone testosterone in sexually mature male frogs as much as tenfold, to levels lower than those in normal female frogs.

"Atrazine-exposed frogs don't have normal reproductive systems," Hayes says. "The males have ovaries in their testes and much smaller vocal organs," which are essential in calling potential mates.

The vocal chords of more than $80 \%$ of male frogs exposed to 1 part per billion (ppb) or more of atrazine were smaller than average; at concentrations of $0.1 \mathrm{ppb}$ and higher, as many as $16 \%$ of the frogs had extra gonads.

Because it has been in use for 40 years in some 80 countries, atrazine's effect on sexual development in male frogs could be a factor contributing to the global decline of amphibians, Hayes says. More than 60 million pounds of atrazine were applied last year in the United States.

The laboratory tests used the African clawed frog (Xenopus laevis), which is very sensitive to hormones that mimic its own sex hormones. If raised in a pond with the female hormone estrogen, for example, all Xenopus tadpoles turn into females. In the presence of male androgens such as testosterone, the frogs grow larger voice boxes.

"Atrazine probably does not have such severe effects on humans, because it does not accumulate in tissue and humans don't spend their lives in water the way frogs do," Hayes says. Nevertheless, the effects of atrazine on frogs could be a sign that the herbicide is subtly affecting human sex hormones.

The U.S. Environmental Protection Agency is currently re-evaluating allowable levels of atrazine in drinking water and the environment. Hayes found hermaphroditism in frogs at levels as low as $0.1 \mathrm{ppb}$, while draft regulations to protect aquatic life limit 4-day exposures to $12 \mathrm{ppb}$. Levels as high as $40 \mathrm{ppb}$ have been measured in rain and spring water in parts of the Midwest, while atrazine in agricultural runoff can be present at several parts per million.

Hayes and his colleagues subsequently visited

\section{Oral exposure to hormones "masculinizes" female finches}

Female zebra finches that orally ingest the hormone estradiol benzoate as chicks develop "masculinized" brains and can sing if stimulated with testosterone as adults, UC Davis scientists have found.

Scientists discovered several decades ago that female finches exposed to estradiol - an estrogen commonly used in hormone replacement therapy - can sing like their male counterparts. Now UC scientists have found that giving doses of hormones to female finch chicks orally, a natural route of exposure to estrogenic chemicals in the environment, can induce "truly significant brain changes," UC Davis animal science professor James Millam says.

Exposure to estradiol benzoate also caused infertility in male finches and hindered the songbirds' ability to reproduce, according to studies by Millam and colleagues published in the December and April issues of Hormones and Behavior.

"Our results indicate that songbird populations may be at risk if they are exposed to estrogenic chemicals as chicks," Millam says.

Hormones are

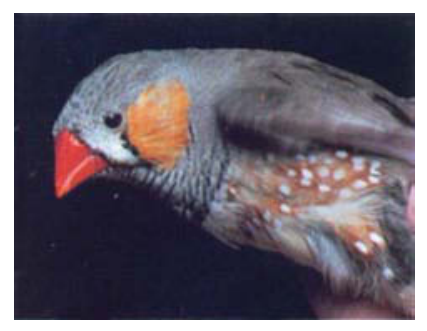

Male zebra finches exposed to estradiol benzoate, an estrogen, had a greater incidence of infertility and reduced reproductive ability. powerful chemicals that regulate sexual development and reproductive ability. Synthetic hormones are leaking into the environment and may 